\title{
Optimization of Parameters of the Kelvin Element in Vibration Analysis
}

\author{
A. Kuznetsov, M. Mammadov, E. Hajilarov \\ School of Information Technology and Mathematical Sciences University of Ballarat \\ Mt. Helen Campus, Victoria Australia \\ e-mail: alexeykuznetsov@s,students.ballarat.edu.au
}

\begin{abstract}
In this paper we consider the problem of finding optimal parameters of the Kelvin element in vibration analysis. This problem is based on finding analytical solution of the initial ODE for development of the optimization model. Such technique allows us to compute optimal parameters of Kelvin element.
\end{abstract}

\section{INTRODUCTION AND LITERATURE REVIEW}

In this paper we consider the problem of finding optimal parameters of the vehicle suspensions given roads with different profiles and different times the driver or passenger is exposed while the vehicle is driven on these roads. The method introduced in this paper consists of the following steps. First, we describe a model suitable for vibration analysis. Then, we find analytical solution of the vibrating model and impose restrictions according to the ISO standard [4]. And, finally, we construct an objective function and search for the global minimum of this function with respect to the spring stiffness and viscosity of the damper of the model. This ensures maximum comfort and safeness for a driver and passenger in a moving vehicle.

Currently, there are quite a lot of publications on modelling vehicle suspensions [1,3] and methods devoted to finding optimal parameters of suspensions at constant harmonic excitations [2,7-11], none of the reviewed works utilize the ISO recommendations on evaluation of the admissible level of vertical accelerations [4]. In the present paper we apply the ISO recommendations to find optimal parameters of suspensions. As far as we aware, no similar investigations have been made in this area in the literature. Such a combination ensures provision of the maximal ride comfort at the long-term exposure to vertical accelerations due vehicle movement along roads with different road profiles. In the last section of the paper we present several numerical examples that demonstrate application of the proposed approach to the construction of suspension.

\section{FORMULATION OF OPTIMIZATION PROBLEM}

\subsection{Kelvin element and its second order equations}

Kelvin element (sometimes called as Voigt element due to another originator) is the main type of viscoelastic elements used in modelling vehicle suspensions, tires, and human muscular-skeletal structure. The principle scheme of such an element is given in Figure 1.

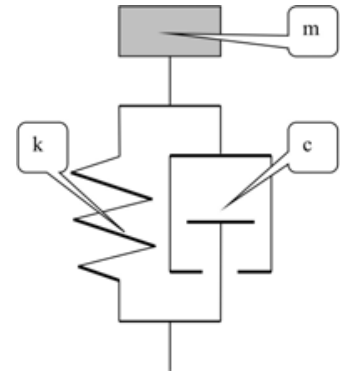

Fig. 1. Kelvin (Voigt) element

It is assumed that springs and dashpots used in constructing the basic viscoelastic elements are weightless and have linear and constant with time response characteristics. The corresponding ODE for the freely vibrating mass is

$$
M \ddot{x}+C \dot{x}+K x=0,
$$

where $x$ is the deflection from the equilibrium position; the mass $M$, the viscosity factor $C$, and the spring rate $K$ are material constants independent of $x, \dot{x}$, and time $t$. The obvious physical reasons imply

$$
M>0, C \geq 0, K \geq 0
$$

\subsection{Deflection of the support and analytical solutions}

Herein, we construct analytical solution for the case when harmonic (vertical) deflection $u(t)=A \exp (i \omega t)$ is applied to the support, where $A$ is amplitude of harmonic deflections of the support. We shall also apply a constant gravity force $M g$ to the mass. The governing second order equation for the considered case is:

$$
M \ddot{x}(t)+C \dot{x}(t)+K x(t)=C \dot{u}(t)+K u(t)-M g
$$

Introducing a new variable $v=\dot{x}$, and denoting $\vec{X}=\left(\begin{array}{l}x \\ v\end{array}\right)$, we transform (3) to the following system of two ODEs of the first order. 


$$
\dot{\vec{X}}=\mathbf{G} \cdot \vec{X}
$$

where

$$
\mathbf{G}=\left(\begin{array}{cc}
0 & 1 \\
-\frac{K}{M} & -\frac{C}{M}
\end{array}\right)
$$

Matrix $\mathbf{G}$ is known as the Jacobian of system (4).

The partial solution of (3) corresponds to the steady state vibrations of the mass of the Kelvin Element. Thus, we can find a partial solution in the form (see, for example, [12]):

$$
\vec{X}(t)=(i \omega \mathbf{I}-\mathbf{G})^{-1} \cdot \vec{P}_{h}
$$

with harmonic loading vector $\vec{P}_{h}$ :

$$
\vec{P}_{h}(t)=\left(\left(\frac{C}{M} \dot{u}(t)+\frac{K}{M} u(t)-g\right) A\right)
$$

Combining (4) - (7) yields deflection $x(t)$ of the mass and its second derivative - acceleration $a(t)=\ddot{x}(t)$ :

$$
a(t)=-i \omega^{2} \frac{(\omega C+i K-i M g)}{\omega^{2} M-i \omega C-K} A e^{i \omega t}
$$

\subsection{Vibration evaluation and Objective Function construction.}

According to the ISO 2631 [4] standards, vibration evaluation includes measurements of the weighted rootmean-square (r.m.s) accelerations.

In ISO 2631 the r.m.s weighted acceleration $a_{w}$ is defined by the following formula:

$$
a_{w}=\left(\frac{1}{T} \int_{0}^{T} a_{w}^{2}(t) d t\right)^{\frac{1}{2}}
$$

where $T$ is a duration of measurement, and $a_{w}(t)$ is the frequency weighted acceleration:

$$
a_{w}(t)=\left(\sum_{i}\left(W_{i} a_{i}(t)\right)^{2}\right)^{\frac{1}{2}} .
$$

In (10) $W_{i}$ is the weighting factor for the $i$-th frequency band $\omega_{i}$ of deflection of Kelvin's element support and $a_{i}(t)$ is the acceleration function for the corresponding frequency band defined by (8). In applications, $W_{i}$ values will be taken form ISO 2631 .

Combining (9) and (10) we obtain the following expression:
$a_{w}=\left(\frac{1}{T} \int_{0}^{T} \sum_{i}\left(W_{i} a_{i}(t)\right)^{2} d t\right)^{\frac{1}{2}}=\left(\sum_{i} W_{i}^{2} A_{i}(T)\right)^{\frac{1}{2}}$

where

$$
A_{i}(T)=\frac{1}{T} \int_{0}^{T} a_{i}^{2}(t) d t
$$

The aim should be to find such parameters of spring stiffness $K$ and damper viscosity $C$ that the r.m.s weighted acceleration $a_{w}$ is minimal. This leads to the following optimization problem:

$$
a_{w}=\left(\sum_{i} W_{i}^{2} A_{i}(T)\right)^{\frac{1}{2}}
$$

Subject to: $K, C \in B$.

Here $B$ is a box defined by $B=\left[K_{\min }, K_{\max }\right] \times\left[C_{\min }, C_{\max }\right] ; K_{\min }, K_{\max }$ are the minimum and maximum values of stiffness of the spring, $C_{\min }, C_{\max }$ are the minimum and maximum values of viscosity of the damper.

As we can see, form (12) and (8), the objective function $a_{w}$ is a function of $K, C$.

\subsection{Steady-state vibrations}

As it was mentioned above, the steady state response corresponds to the partial solution of (3). For steady statestate vibrations the problem of minimization (13) can be derived in the following manner using only the real part of the acceleration (8) for each considered $i$-th band of excitation:

$$
\operatorname{Re}\left(a_{i}(t)\right)=A_{i} L_{i} \cos \left(\omega_{i} t\right)+A_{i} Q_{i} \sin \left(\omega_{i} t\right)
$$

where $A_{i}$ is an amplitude of harmonic deflections of the support corresponding to $i$-th frequency band and

$$
\begin{aligned}
L_{i} & =\frac{\omega_{i}^{2}\left(\left(M \omega_{i}^{2}-K\right)(K-M g)-C^{2} \omega_{i}^{2}\right)}{C^{2} \omega_{i}^{2}+\left(M \omega_{i}^{2}-K\right)} \\
Q_{i} & =-\frac{C \omega_{i}^{3}\left(M \omega_{i}^{2}-M g\right)}{C^{2} \omega_{i}^{2}+\left(M \omega_{i}^{2}-K\right)}
\end{aligned}
$$

Therefore, using (15) we can transform (9) as follows: 
$A_{i}(T)=\frac{A_{i}^{2} L_{i}^{2}}{2 T}\left(\frac{\cos \left(\omega_{i} T\right) \sin \left(\omega_{i} T\right)}{\omega_{i}}+T\right)+\frac{A_{i}^{2} L_{i} Q_{i}}{T}\left(-\frac{\cos ^{2}\left(\omega_{i} T\right)}{\omega_{i}}\right)+$ $+\frac{A_{i}^{2} Q_{i}^{2}}{2 T}\left(\frac{\cos \left(\omega_{i} T\right) \sin \left(\omega_{i} T\right)}{\omega_{i}}+T\right)$

In (17), the terms corresponding to $\sin \left(\omega_{i} T\right)$ and $\cos \left(\omega_{i} T\right)$ converge to zero as $T \rightarrow \infty$. Therefore, for large $T, A_{i}(T)$ will be mostly dependent on the other terms and that allows us to consider only the limits $A_{i}(T)$ as $T \rightarrow \infty$. We have:

$$
\lim _{T \rightarrow \infty} A_{i}(T)=\frac{A_{i}^{2}}{2}\left(L_{i}^{2}+Q_{i}^{2}\right) .
$$

Thus, the objective function (13) can be simplified by formula (18) and represented in the following manner:

$$
F(K, C)=\left[\sum_{1}^{n}\left(\frac{A_{i}^{2}}{2}\left(L_{i}^{2}+Q_{i}^{2}\right) W_{i}^{2}\right)\right]^{\frac{1}{2}}
$$

where $n$ is the number of frequencies in vibration spectrum and again, $L_{i}, Q_{i}$ are defined by (16). Thus, Problem (13),(14) can be represented as the follows:

$$
\begin{aligned}
& \text { Minimize: } F(K, C) \text {. } \\
& \text { Subject to: } K, C \in B \text {. }
\end{aligned}
$$

We note that, this statement involves steady solutions only and, in contrast of (13),(14), it does not use $T$.

\subsection{Vibrations of the Kelvin Element on different sequential} excitations of the support

Now, consider the case with several sequential vibration periods $T_{k},(k=1, m)$ to different magnitudes and durations of Kelvin Element. According to ISO 2631, the following formula is applied to calculate the total comfort corresponding to all periods $T_{k}$.

$$
\tilde{F}(K, C)=\left[\frac{\sum_{1}^{k}\left(T_{k} a_{w k}^{2}\right)}{\sum_{1}^{k} T_{k}}\right]^{\frac{1}{2}}
$$

where $a_{w k}$ is the vibration magnitude for exposure period $T_{k}$. In terms of steady solutions it can be represented as $a_{w k}=F(K, C)$ in (19):

$$
a_{w k}=\left[\sum_{1}^{n_{k}}\left(\frac{A_{i k}^{2}}{2}\left(L_{i k}^{2}+Q_{i k}^{2}\right) W_{i k}^{2}\right)\right]^{\frac{1}{2}} .
$$

Index $k$ indicates the corresponding $k$-th period in of vibration of the Kelvin element and $L_{i k}, Q_{i k}$ are defined similar to (16) as follows:

$$
\begin{aligned}
L_{i k} & =\frac{\omega_{i}^{2}\left(\left(M \omega_{i k}^{2}-K\right)(K-M g)-C^{2} \omega_{i k}^{2}\right)}{C^{2} \omega_{i k}^{2}+\left(M \omega_{i k}^{2}-K\right)} \\
Q_{i k} & =-\frac{C \omega_{i k}^{3}\left(M \omega_{i k}^{2}-M g\right)}{C^{2} \omega_{i k}^{2}+\left(M \omega_{i k}^{2}-K\right)}
\end{aligned} .
$$

Thus, objective function (22) leads to the following optimization problem:

$$
\begin{aligned}
& \text { Minimize: } F(K, C) \\
& \text { Subject to: } K, C \in B .
\end{aligned}
$$

This is an optimization problem with box constraints (26). The objective function (23) is not convex or concave, therefore the problem (25) may have many local solutions. We are looking for a global solution in our research. In the calculations below, we use the global optimization algorithm AGOP, introduced in $[5,6]$.

\section{NUMERICAL EXAMPLES}

\subsection{Single spectrum vibration}

Here we assume that the mass $M$ is constant and the deflection of the Kelvin element is defined by a periodic function given in (7). It is also assumed that the frequency of deflections $\omega_{i}$ and the amplitude $A_{i}$ are constant during the vibrations and the numbers of bands of the total vibration spectrum are $n=6,5,4$. The weighting factors $W_{i}$ corresponding each $\omega_{i}$ were taken from the ISO 2631. The spring stiffness here is $K \in[200,1500]$ and damper viscosity is $C \in[1,10]$. Looking at (14), we can represent $B=[200,1500] \times[1,10]$.

The parameters of Kelvin element and the bands of vibrating spectrum are taken as follows: 
$M=1 \mathrm{~kg}$,

$$
\begin{aligned}
& \omega_{1}=1 H z, \quad A_{1}=0.08 m, \quad W_{1}=0.482 \\
& \omega_{2}=2 \mathrm{~Hz}, \quad A_{2}=0.08 m, \quad W_{2}=0.531 \\
& \omega_{3}=5 \mathrm{~Hz}, \quad A_{3}=0.002 \mathrm{~m}, W_{3}=1.039 \\
& \omega_{4}=8 \mathrm{~Hz}, \quad A_{4}=0.002 \mathrm{~m}, W_{4}=1.036 \\
& \omega_{5}=4 \mathrm{~Hz}, \quad A_{5}=0.001 \mathrm{~m}, W_{5}=0.967 \\
& \omega_{6}=3 \mathrm{~Hz}, \quad A_{6}=0.0005 \mathrm{~m}, W_{6}=0.804
\end{aligned}
$$

Some additional values for bands will also be considered for better performance and comparability.

The corresponding computational results for the acceleration of mass for the considered model are presented in Table I.

Analyzing the presented results, we can find relationships between optimal values of the Kelvin element and its different excitations. We can find the tendency that could be expected: in terms of optimality, high stiffness of spring requires low damping and high viscosity of damper requires low stiffness of spring. However, even in this simple case, different amplitudes of vibrations lead to different optimal values $K, C$.

While considering different sequential vibrations, the problem of finding optimal values $K, C$ might be quite

\begin{tabular}{|c|c|c|c|c|}
\hline $\begin{array}{c}\text { Additional } \\
\text { amplitude } \\
\text { values of } \\
\text { excitation of } \\
\text { the } \\
\text { support } A_{i}\end{array}$ & $\begin{array}{c}\text { Frequency of } \\
\text { variable } \\
\text { amplitudes } \boldsymbol{\omega}_{i}\end{array}$ & $\begin{array}{c}\text { Optimal } \\
\text { Damper } \\
\text { viscosity } \\
C\end{array}$ & $\begin{array}{c}\text { Optimal } \\
\text { Stiffness of } \\
\text { spring } K\end{array}$ & $\begin{array}{l}\text { The minimum value } \\
\text { of Objective Function }\end{array}$ \\
\hline \multicolumn{2}{|c|}{$n=6$} & & & \\
\hline$A_{6}$ & $\omega_{6}$ & & & \\
\hline 0.0005 & 3 & 3.1826 & 283.186 & 3.2828 \\
\hline 0.0004 & 3 & 2.5065 & 287.2385 & 3.2459 \\
\hline 0.0006 & 3 & 3.7643 & 278.7984 & 3.3184 \\
\hline \multicolumn{2}{|c|}{$n=5$} & & & \\
\hline$A_{5}$ & $\omega_{5}$ & & & \\
\hline 0.001 & 4 & 1 & 302.2257 & 3.1315 \\
\hline 0.002 & 4 & 7.8 & 200 & 3.5049 \\
\hline 0.005 & 4 & 6.8890 & 200 & 4.2166 \\
\hline \multicolumn{2}{|c|}{$n=4$} & & & \\
\hline$A_{4}$ & $\omega_{4}$ & & & \\
\hline 0.001 & 8 & 7.5456 & 200 & 0.8667 \\
\hline 0.0011 & 8 & 7.3676 & 200 & 0.8896 \\
\hline 0.0012 & 8 & 1 & 281 & 0.9028 \\
\hline
\end{tabular}
complicated. In the following example we are considering such a case.

TABLE I

THE OPTIMAL PARAMETERS C AND K FOR DIFFERENT SINGLE VIBRATION SPECTRUM IN EXAMPLE 1

\subsection{Multiple spectrum vibration.}

Here we adopt all the notations mentioned in Section 3.1 and consider two different vibration spectra that consist of $n_{1}=n_{2}=4$ number of bands each and set the time of vibrating periods on each spectrum as $T_{1}$ and $T_{2}$.

The parameters of Kelvin element and the bands of vibrating spectra are taken as follows:

the first spectrum:

$M=1 \mathrm{~kg}$,

$$
\begin{array}{lll}
\omega_{11}=2 H z, & A_{11}=0.08 m, & W_{11}=0.531 \\
\omega_{21}=4 H z, & A_{21}=0.04 m, & W_{21}=0.967 \\
\omega_{31}=25 H z, & A_{31}=0.001 m, & W_{31}=0.513 \\
\omega_{41}=8 H z, & A_{41}=0.001 m, & W_{41}=1.036
\end{array}
$$

and the second vibrating spectrum:

$$
\begin{array}{lll}
\omega_{12}=2 H z, & A_{12}=0.02 m, & W_{12}=0.531 \\
\omega_{22}=4 H z, & A_{22}=0.001 m, & W_{22}=0.967 \\
\omega_{32}=25 H z, & A_{32}=0.001 m, & W_{32}=0.513 \\
\omega_{42}=8 H z, & A_{42}=0.005 m, & W_{42}=1.036
\end{array}
$$

The computational results for the acceleration of mass for the considered model are presented in Table II with different proportions between periods $T_{1}, T_{2}$ spending on each vibration spectra.

Looking at the results, we can see the tendencies of changing optimal values of spring and damper of the Kelvin element in terms of different proportions of the vibrating periods: the vibration periods $T_{1}, T_{2}$ influence on optimal values $K, C$. For example, when $T_{1} \gg T_{2}$, it could be more preferable to have in the Kelvin element $C_{\max }$ and $K=461.3286$, at the same time when $T_{1}<<T_{2}$, low vibrations achieves at $C_{\min }$ and $K=400.5220$.

TABLE II

THE OPTIMAL PARAMETERS C AND K FOR DIFFERENT TIME PERIODS T $\mathrm{T}_{1}$ AND $\mathrm{T}_{2}$ IN EXAMPLE 2

\begin{tabular}{|c|c|c|c|}
\hline $\begin{array}{c}\text { Proportions between vibrating } \\
\text { periods }\end{array} T_{1}, T_{2}$ & $\begin{array}{c}\text { Optimal } \\
\text { Damper } \\
\text { viscosity }\end{array}$ & $\begin{array}{c}\text { Optimal } \\
\text { Stiffness of } \\
\text { spring } K\end{array}$ & $\begin{array}{c}\text { The minimum } \\
\text { value of objective } \\
\text { Function }\end{array}$ \\
\hline$T_{1}=\frac{1}{2} ; T_{2}=\frac{1}{2}$ & 5.3892 & 423.3512 & 6.0683 \\
\hline$T_{1}=\frac{4}{5} ; T_{2}=\frac{1}{5}$ & & & \\
\hline$T_{1}=\frac{1}{5} ; T_{2}=\frac{4}{5}$ & & 461.3286 & \\
\hline
\end{tabular}


Also we see that the optimal values of $K$ doesn't change much with different proportions of vibration periods, and $C$ changes from minimum values to maximum values of viscosity.

\section{REFERENCES}

1. Chen P.C., Huang A.C., Adaptive Multi-surface Sliding Control of Hydraulic Active Suspension Systems, Journal of Vibration and Control, 2005, No.11

2. Georgiou G., Verros G., Natsiavas S., Multi-objective Optimization of Quarter-car Models with a Passive or Semi-active Suspension System, Vehicle System Dynamics,2007, vol.45, No.1, pp. 77-92.

3. Harris' Shock and Vibration Handbook, $5^{\text {th }}$ edition, Editors C.M. Harris and A.G. Piersol, McGraw-Hill, N.Y., 2002.

4. ISO 2631-1:1997, "Mechanical Vibration and Shock - Evaluation of human exposure to whole-body vibration - Part 1".

5. Mammadov, M. A., Rubinov, A. M., Yearwood, J.: Dynamical systems

described by relational elasticities with applications to global optimization. In: V. Jeyakumar and A. Rubinov (Eds.) Continuous Optimisation: Current Trends and Applications, Springer, 2005, pp. 365-387.

6. Mammadov, M.A.: A new global optimization algorithm based on dynamical

systems approach. In: A. Rubinov and M. Sniedovich (eds) 6th International

Conference on Optimization: Techniques and Applications, Ballarat, Australia, 2004.

7. Metallidis P., Verros G., Natsiavas S., Fault Detection and Optimal Sensor Location in Vehicle Suspensions, Journal of Vibration and Control, 2003, No.9

8. Papalukopoulos C., Giadopulos D., Natsiavas S., Dynamics of Large Scale Vehicle Models Coupled with Driver Biodynamic Models, $5^{\text {th }}$ GRACM International Congress on Computational Mechanics, Limassol, 2005.

9. Papalukopoulos C., Theodosiou C., Natsiavas S., Nonlinear Dynamics of Vehicle Models Coupled with Biodynamic Passenger Models, $2^{\text {nd }}$ International Conference on Nonlinear Normal Modes and Localization in Vibrating Systems, Samos, 2006.

10. Verros G., Goudas H., Natsiavas S., Dynamics of Large Scale Vehicle Models Using ADAMS/FLEX, International ADAMS User Conference, 2000.

11. Verros G., S. Nastiavas S., Papadimitriou C., Design Optimization of Quarter-car Models with Passive and Semi-active Suspensions under Random Road Excitation, Journal of Vibration and Control, 2005, No. 1, pp. 581-606.

12. Zwillinger D., Handbook of Differential Equations. $3^{\text {rd }}$ edition, Academic Press, N.Y., 1997. 